\title{
Submolecular-scale Investigations on metal-phthalocyanine monolayers by frequency modulation atomic force microscopy
}

\section{$\operatorname{AUTHOR}(\mathrm{S}):$}

Ichii, Takashi; Fukuma, Takeshi; Yoda, Tadashi; Kobayashi, Kei; Matsushige, Kazumi; Yamada, Hirofumi

\section{CITATION:}

Ichii, Takashi ...[et al]. Submolecular-scale Investigations on metal-phthalocyanine monolayers by frequency modulation atomic force microscopy. Journal of Applied Physics 2010, 107(2): 024315.

\section{ISSUE DATE:}

2010-01-15

URL:

http://hdl.handle.net/2433/147209

\section{RIGHT:}

Copyright 2010 American Institute of Physics. This article may be downloaded for personal use only. Any other use requires prior permission of the author and the American Institute of Physics. The following article appeared in JOURNAL OF APPLIED PHYSICS107, 024315 (2010) and may be found at 


\title{
Submolecular-scale Investigations on metal-phthalocyanine monolayers by frequency modulation atomic force microscopy
}

\author{
Takashi Ichii, ${ }^{1, a)}$ Takeshi Fukuma, ${ }^{1, b)}$ Tadashi Yoda, ${ }^{1}$ Kei Kobayashi, ${ }^{2}$ Kazumi Matsushige, ${ }^{1}$ \\ and Hirofumi Yamada ${ }^{1, c)}$ \\ ${ }^{1}$ Department of Electronic and Engineering, Kyoto University, Katsura, Nishikyo, Kyoto 615-8510, Japan \\ ${ }^{2}$ Innovative Collaboration Center, Kyoto University, Katsura, Nishikyo, Kyoto 615-8520, Japan
}

(Received 5 August 2009; accepted 4 December 2009; published online 29 January 2010)

Copper-phthalocyanine $(\mathrm{CuPc})$ monolayers and cobalt-phthalocyanine monolayers deposited on $\mathrm{Au}(111)$ surfaces were investigated by frequency modulation atomic force microscopy (FM-AFM). Submolecular-resolution topographic images were successfully obtained for both samples. Despite the similar molecular geometry of the two molecules, they showed clearly different contrasts in the topographic images. The origin of the contrast is discussed in terms of the relationship of the molecular orbitals and the chemical interaction between the tip and the molecules. In addition, a molecular-resolution surface potential (SP) image was obtained on $\mathrm{CuPc}$ monolayers using Kelvin probe force microscopy (KFM) utilizing FM-AFM. The molecular-scale SP contrast was explained by the electric dipole moment at the organic/metal interface. This result suggested the possibility of the detection of the single molecular dipole moment by KFM. (C) 2010 American Institute of Physics. [doi:10.1063/1.3284094]

\section{INTRODUCTION}

Electronic devices using organic semiconductors, such as field effect transistors, electroluminescence devices, and solar cells, have been studied by many research groups because they have numerous attractive advantages such as easy fabrication and mechanical flexibility. The performance of organic devices is determined not only by the molecular species but also by the molecular arrangement and the electronic states of the molecules close to the electrodes. ${ }^{1,2}$ Therefore, studies on the structures and electrical properties of organic monolayers formed on metal substrates will give us important information to improve the performance of organic electronic devices.

Frequency modulation atomic force microscopy (FMAFM), which is also known as noncontact atomic force microscopy, has been developed to investigate various surface structures at the true-atomic resolution, such as semiconductors, metals, and even insulators. ${ }^{3}$ It has been also applied to molecular-resolution imaging of organic molecules. The imaging mechanisms of FM-AFM have also been studied from the experimental and theoretical points of view. Several reports suggested that the short-range chemical interactions between a tip front atom and a sample atom is a major origin of atomic-scale contrast. ${ }^{3,4}$ However, these discussions on imaging mechanisms have been mainly focused on inorganic materials and there are few reports on organic materials.,

FM-AFM also allows visualization of the distributions of various material properties by detecting diverse tip-tosample interactions, such as chemical and electrostatic forces. In particular, Kelvin probe force microscopy (KFM)

\footnotetext{
${ }^{\text {a) }}$ Present address: Department of Materials Science and Engineering, Kyoto University, Yoshida-Honmachi, Sakyo, Kyoto 606-8501, Japan.

${ }^{b}$ Present address: Frontier Science Organization, Kanazawa University, Kakuma-cho, Kanazawa, 920-1192, Japan.

${ }^{c)}$ Electronic mail: h-yamada@kuee.kyoto-u.ac.jp.
}

utilizing FM-AFM is an outstanding method for investigating surface potential (SP) distribution. ${ }^{7}$ The SP of organic thin films reflects dipole moments of the molecules and those on interfaces between the molecules and substrates, ${ }^{1}$ which is important information for developing organic electronic devices.

In this study, we investigated metal-phthalocyanine (MPc) monolayers formed on $\mathrm{Au}(111)$ surfaces using FMAFM and KFM. MPcs are well known as typical molecules with $\pi$-conjugated systems and they have been applied to organic devices. ${ }^{8-10}$ The structure of MPc is shown schematically in Fig. 1. MPc consists of four iminoisoindoline units with a metal ion in the center of the molecule. It has a widely delocalized $\pi$-electron orbital sticking out of the molecular plane, which can be modified by changing the central metal ion. We chose copper-phthalocyanine ( $\mathrm{CuPc}$ ) and cobaltphthalocyanine $(\mathrm{CoPc})$ among various MPcs because they have completely planar structures. Topography of the CuPc and $\mathrm{CoPc}$ monolayers was investigated by FM-AFM and

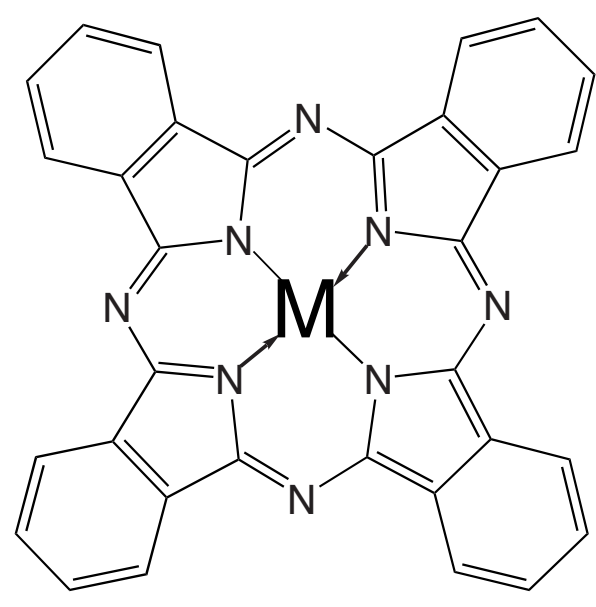

FIG. 1. Molecular structure of MPc. 
their submolecular-scale contrasts were compared in order to elucidate the formation mechanism of submolecular-scale contrasts in FM-AFM. SP distribution of a CuPc monolayer on the $\mathrm{Au}(111)$ surface was also investigated by KFM and the possibility of detecting single molecular dipole moment was examined.

\section{EXPERIMENTAL DETAILS}

All the experiments were performed in an ultrahigh vacuum (UHV) environment. FM-AFM images were obtained with the constant-amplitude mode. A commercially available UHV-AFM instrument (JEOL: JSPM-4500) was used with some modifications. The original FM demodulator was replaced with a home-built FM detector. ${ }^{11}$ All the images in this report were obtained by constant frequency shift $(\Delta f)$ mode. Highly doped Si cantilevers (Nanoworld: $\mathrm{NCH}$ ) were used. The typical spring constant and resonance frequency of the cantilevers were $40 \mathrm{~N} \mathrm{~m}^{-1}$ and $300 \mathrm{kHz}$, respectively. The typical measured Q-factor of the cantilevers in UHV was 30 000. The estimated cantilever vibration amplitude was about $10 \mathrm{~nm}_{\mathrm{p}-\mathrm{p}}$. SP measurements were made by KFM using the FM detection method. ${ }^{7}$ A modulation bias voltage $\left(600 \mathrm{~Hz}, 10 \mathrm{~V}_{\mathrm{p}-\mathrm{p}}\right)$ was applied to the sample.

CuPc powder (Tokyo Kasei Kogyo. Co., Ltd) was purified by sublimation before use. CoPc powder that was already purified by sublimation was bought from Tri Chemical Laboratories Inc. The Au(111) surfaces were prepared by evaporating gold onto freshly cleaved mica substrates at a substrate temperature of $430{ }^{\circ} \mathrm{C}$ in a vacuum (about 1.0 $\times 10^{-7}$ Torr). The thickness of the gold films was about 250 $\mathrm{nm}$. The Au substrates were transferred to the UHV chamber via atmosphere and heated at $200{ }^{\circ} \mathrm{C}$ for $1 \mathrm{~h}$ to eliminate impurities. Then, they were cooled down to room temperature. $\mathrm{CuPc}$ and $\mathrm{CoPc}$ molecules were deposited from a Knudsen at about 6-7 layers/h and 2-3 layers/h, respectively. After deposition, they were transferred from the deposition chamber to the AFM sample stage without being exposed to the air.

\section{RESULTS AND DISCUSSION}

\section{A. Submolecular-scale topographic imaging}

Figure 2(a) shows a submolecular-resolution topographic image of a $\mathrm{CuPc}$ monolayer on the $\mathrm{Au}(111)$ surface. The $\mathrm{CuPc}$ molecules formed square-lattice structures with a lattice constant of approximately $1.3 \mathrm{~nm}$ and each of them was imaged like a four-leaf clover, which corresponds to the molecular structure shown in Fig. 1. An outline of a molecule is drawn in Fig. 2(a). The central part of the CuPc molecules was imaged as an apparent hole. The height difference between the holes and the Pc rings was about 40-60 pm. The four iminoisoindoline units around the hole had the same height, which indicate the $\mathrm{CuPc}$ molecules were lying flat on the $\mathrm{Au}(111)$ surface. Previously, FM-AFM images of $\mathrm{CuPc}$ monolayers on $\mathrm{MoS}_{2}$ surfaces with submolecular-scale contrasts were reported, where the $\mathrm{CuPc}$ molecules were slightly inclined to the substrate surface plane. ${ }^{12}$ This structural difference should be caused by the difference in the interaction of $\mathrm{CuPc}-\mathrm{Au}(111)$ and that of $\mathrm{CuPc}-\mathrm{MoS}_{2}$.

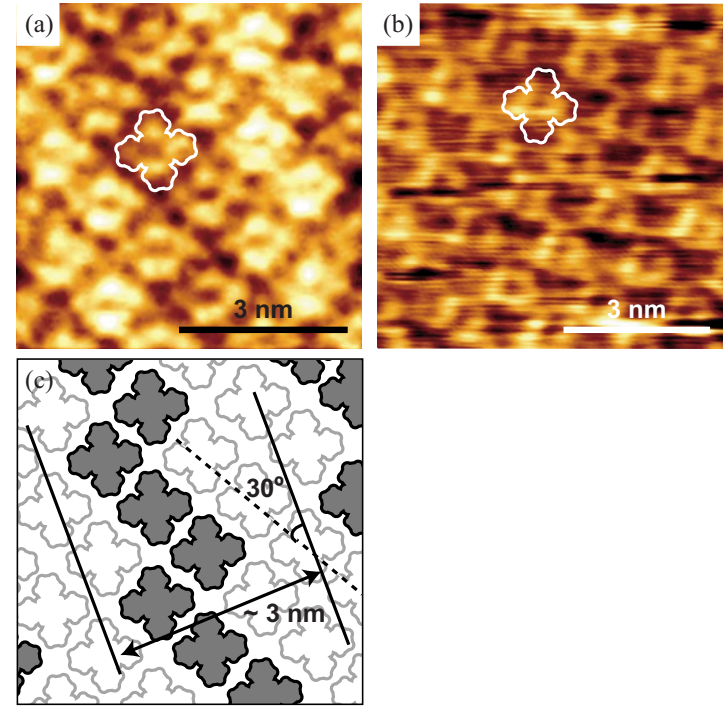

FIG. 2. (Color online) Topographic images of CuPc monolayers on $\mathrm{Au}(111)$ surfaces obtained by FM-AFM. (a) $6 \times 6 \mathrm{~nm}^{2}, \Delta f=-450 \mathrm{~Hz}$. (b) 7 $\times 7 \mathrm{~nm}^{2}, \Delta f=-134 \mathrm{~Hz}$. (c) An illustration of molecular arrangements in (a).

Figure 2(b) shows another submolecular-resolution topographic image of a $\mathrm{CuPc}$ monolayer on an $\mathrm{Au}(111)$ surface. This image was taken on a different film and using a different tip. An outline of a molecule is drawn in Fig. 2(b). The central part of the CuPc molecules was again imaged as a hole. The height difference between the hole and the Pc ring was about 30-40 pm in this case, which was almost the same as that measured in Fig. 2(a). The depression in the center of the molecules was also observed on the multilayer film of $\mathrm{CuPc}$ on the $\mathrm{Au}(111)$ surfaces (constant height mode) and $\mathrm{CuPc}$ monolayers on $\mathrm{MoS}_{2}(0001)$ surfaces (constant $\Delta f$ mode). ${ }^{12,13}$ The height difference between the holes and the Pc rings in $\mathrm{CuPc}$ monolayers deposited on $\mathrm{MoS}_{2}$ surfaces was reported as 50-60 pm. Considering these experimental results, it can be concluded that the central part of $\mathrm{CuPc}$ molecules is imaged as a hole with a depth of a few tens of picometers independent of the substrate species and the tip conditions.

It should be noted that stripes originating from the height variation of the CuPc molecules were found in Fig. 2(a). Figure 2(c) shows an illustration of the molecular arrangements in the film in Fig. 2(a). The stripe width was about 3 $\mathrm{nm}$ and they were rotated from the direction of $\mathrm{CuPc}$ molecular rows by about $30^{\circ}$. The width was nearly equal to that of the reconstructed structures of $\mathrm{Au}(111)-22 \times \sqrt{3}$ structures. ${ }^{14}$ Thus, these stripes could be caused by the underlying reconstructed structures of the $\mathrm{Au}(111)$ substrate. This result agreed well with the previous study on CuPc monolayers on $\mathrm{Au}(111)$ surfaces using scanning tunneling microscopy (STM). ${ }^{15}$

Figure 3 shows topographic images of a $\mathrm{CoPc}$ monolayer on an $\mathrm{Au}(111)$ surface. Figure 3(a) shows a large-scale topographic image. Many domain boundaries were clearly observed as dark lines, and many of them bent with an angle of about $60^{\circ}$. Figure 3(b) shows a molecularly resolved topographic image obtained from a part of Fig. 3(a). The CoPc molecules formed quasihexagonally packed structures, 

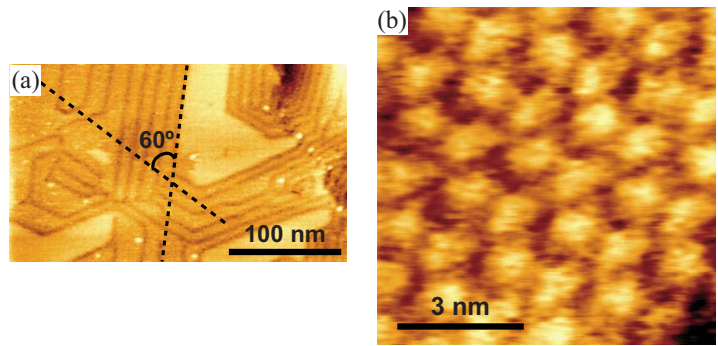

FIG. 3. (Color online) Topographic images of a CoPc monolayer on a $\mathrm{Au}(111)$ surface obtained by FM-AFM. (a) $300 \times 180 \mathrm{~nm}^{2}, \Delta f=-15 \mathrm{~Hz}$. (b) $8 \times 8 \mathrm{~nm}^{2}, \Delta f=-380 \mathrm{~Hz}$.

which are clearly different from the molecular arrangements observed on the $\mathrm{CuPc}$ monolayers in this study. The domain boundaries shown in Fig. 3(a) should reflect the quasihexagonally packed structures. Submolecular resolution was not achieved in these regions while it was achieved on the square lattice of the $\mathrm{CoPc}$ monolayers as described below. Considering these results, it was speculated that the CoPc molecules in the quasihexagonal lattice were considerably inclined from the substrate.

Figure 4(a) shows a large-scale topographic image of a $\mathrm{CoPc}$ monolayer obtained on a different area from Fig. 3(a). In this area, some domain boundaries bent at an angle of about $90^{\circ}$ as observed in the dotted white circle. Figure 4(b) shows a submolecular-resolution image of the CoPc monolayer obtained in this area. An outline of a molecule is drawn in Fig. 4(b). Individual CoPc molecules with a four-leaf clover structure were clearly seen. Here, the CoPc molecules were almost lying flat on the substrate and formed squarelattice structures like the $\mathrm{CuPc}$ molecules in Fig. 2. The domain boundaries bent at about $90^{\circ}$ in Fig. 4(a) reflected these square-lattice structures. The central part of the CoPc molecules was not imaged as a hole unlike the $\mathrm{CuPc}$ molecules. Figure 4(c) shows a submolecular-resolution topographic image obtained from another region of the $\mathrm{CoPc}$ monolayer. This image also showed the clear four-leaf clover structures without holes in the center of the molecules.
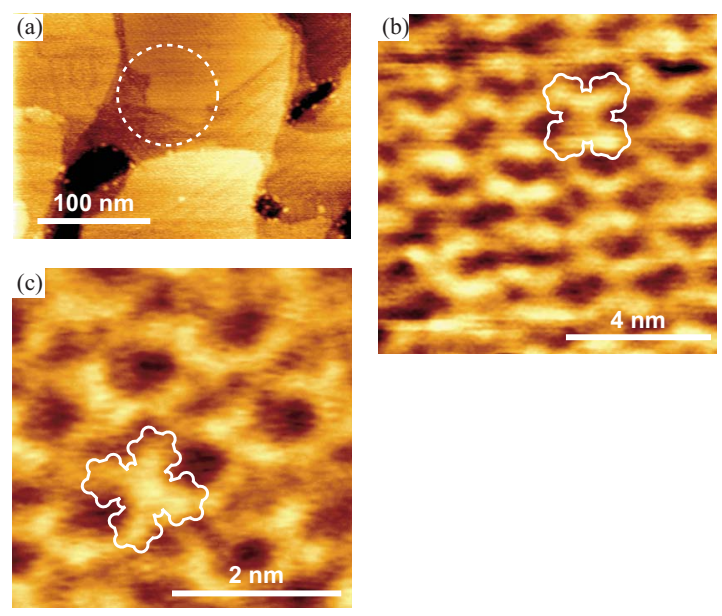

FIG. 4. (Color online) Topographic images of a CoPc monolayer on an $\mathrm{Au}(111)$ surface obtained by FM-AFM. (a) $300 \times 210 \mathrm{~nm}^{2}, \Delta f=-20 \mathrm{~Hz}$. (b) $7 \times 7 \mathrm{~nm}^{2}, \Delta f=-260 \mathrm{~Hz}$. (c) $4 \times 4 \mathrm{~nm}^{2}, \Delta f=-320 \mathrm{~Hz}$.

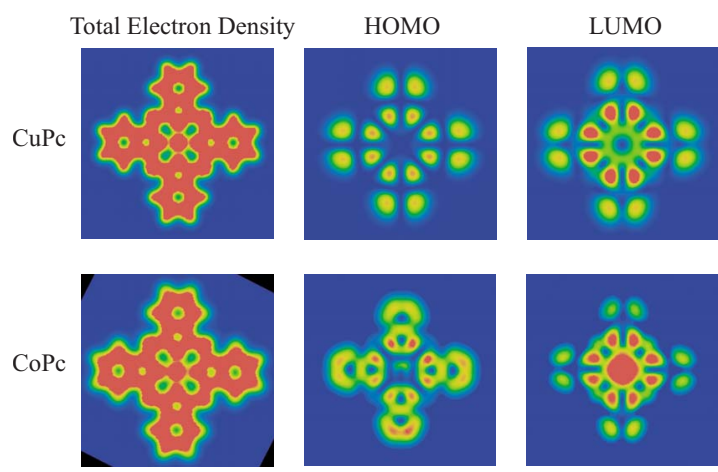

FIG. 5. (Color online) Two-dimensional maps of the total electron density distributions, the electron density distributions of the HOMO, and those of the LUMO at the surface located $0.25 \mathrm{~nm}$ above the molecular planes of $\mathrm{CuPc}$ and $\mathrm{CoPc}$ molecules, calculated based on the density functional theory.

Since $\mathrm{CuPc}$ and $\mathrm{CoPc}$ molecules have completely planar structures and their central metal ions have almost the same ionic radii $\left(57 \mathrm{pm}\right.$ for $\mathrm{Cu}^{2+}$ and $58 \mathrm{pm}$ for $\left.\mathrm{Co}^{2+}\right),{ }^{16}$ the difference in the submolecular-scale contrast between $\mathrm{CuPc}$ and $\mathrm{CoPc}$ cannot be explained by their difference in molecular geometry. In the STM images of the same molecules on the $\mathrm{Au}(111)$ surfaces, the center of $\mathrm{CuPc}$ molecules was imaged as a hole while that of $\mathrm{CoPc}$ molecules was imaged as a protrusion. ${ }^{17,18}$ The formation mechanism of the STM contrasts were explained by the spatial distributions of the molecular orbitals, where d-orbitals of the Co atom contribute to the highest occupied molecular orbital (HOMO) and the lowest unoccupied molecular orbital (LUMO) while those in the $\mathrm{Cu}$ atom in $\mathrm{CuPc}$ do not.

We also calculated the spatial distribution of the electron density and the molecular orbitals of $\mathrm{CuPc}$ and $\mathrm{CoPc}$ molecules based on the density functional theory using MATERIALS STUDIO (Accelrys) with DMOL3 package. Figure 5 shows two-dimensional maps of the total electron density distributions, electron density distributions of the HOMO, and those of LUMO at the surface located $0.25 \mathrm{~nm}$ above the molecular planes of $\mathrm{CuPc}$ and $\mathrm{CoPc}$ molecules. Since the difference in the total electron density between $\mathrm{CuPc}$ and $\mathrm{CoPc}$ was not found, we consider that it does not contribute to the submolecular-scale FM-AFM contrasts. On the other hand, there are clear differences in the electron density of the HOMO and LUMO, especially in those of the LUMO, in which the electron density is higher at the location of Co atom than the surrounding iminoisoindoline units while it is not in the case of $\mathrm{CuPc}$ molecule. The distributions of these molecular orbitals correspond well with the obtained FMAFM contrasts. Therefore we conclude that the electron distributions at the HOMO and LUMO levels are playing important roles in the submolecular-scale contrasts in the FMAFM imaging on organic materials.

It has been theoretically pointed out that hybridization of the electron orbitals between a tip front atom and a surface atom plays an important role in formation of the atomic-scale FM-AFM contrasts on $\mathrm{Si}(111)$ surface imaged by a $\mathrm{Si}$ tip. ${ }^{19}$ In addition, Tagami et al. performed theoretical calculation of interaction between a $\mathrm{C}_{60}$ molecule tip and a $\mathrm{Si}(001)$ surface. They reported that the interaction between the dangling 

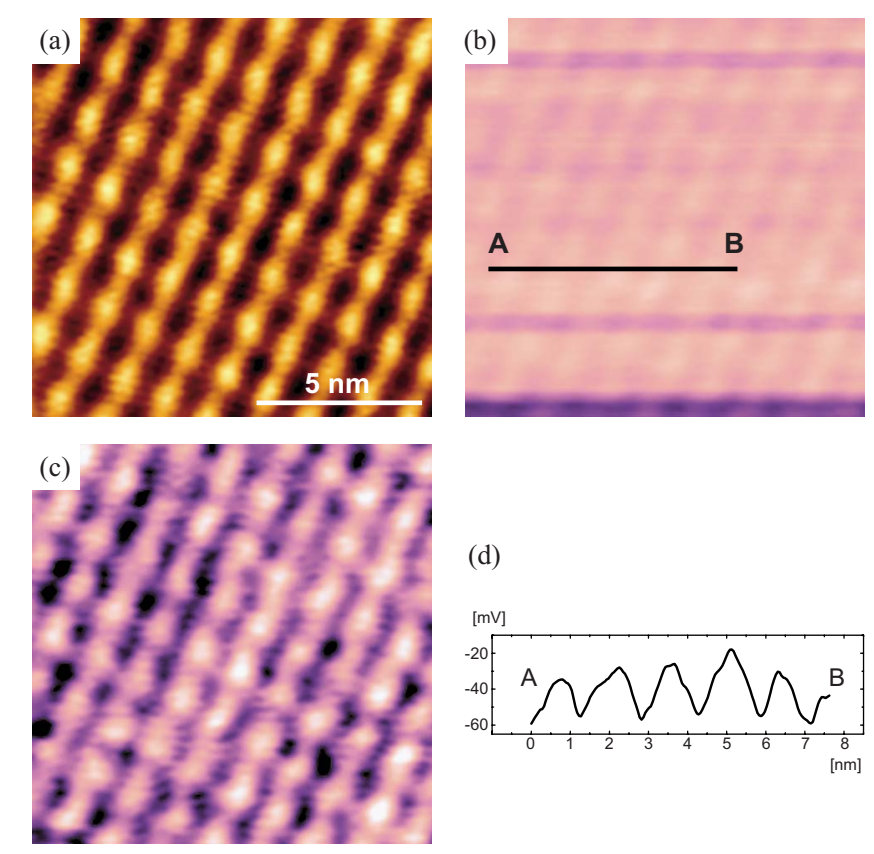

(d)

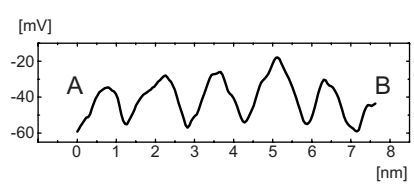

(e)

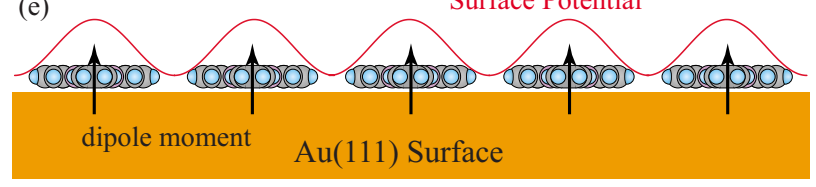

FIG. 6. (Color online) FM-AFM images of a CuPc monolayer on an $\mathrm{Au}$ (111) surface, $12 \times 12 \mathrm{~nm}^{2}, \Delta f=-170 \mathrm{~Hz}$. (a) Topographic image. (b) SP image. (c) Fluctuation-corrected SP image. (d) A cross-sectional profile measured along the A-B line indicated in (b). (e) A model to explain the molecular-scale SP contrast.

bond states of the Si surface and the HOMO and LUMO of the $\mathrm{C}_{60}$ mainly contribute to the short-range force. ${ }^{20}$ These theoretical studies support our conclusion.

\section{B. Molecular-scale surface potential contrast}

Figure 6(a) shows a topographic image of a CuPc monolayer on the $\mathrm{Au}(111)$ surface. Although submolecular resolution was not achieved in this case, a molecularly resolved image was successfully obtained and square lattices with a period of approximately $1.3 \mathrm{~nm}$ were recognized. Figure 6(b) shows an SP image obtained simultaneously with Fig. 6(a) using KFM. There were several horizontal stripes due to some abrupt SP shift. These changes must be because of the abrupt SP shift of the tip, which accompanied some changes in the tip structure. Figure 6(c) shows the SP image after correction of the SP fluctuation in Fig. 6(b). This image clearly showed the molecular-scale SP contrast. The SP difference between the CuPc molecules and the intermolecular region was approximately $30 \mathrm{mV}$, as shown in Fig. 6(d), which shows a cross-sectional profile measured along the A-B line indicated in Fig. 6(b). This result can be explained by the electric dipole moment on the interface between the $\mathrm{CuPc}$ molecules and the Au substrate as illustrated schematically in Fig. 6(e). It is unclear whether the measured SP variation is quantitative or not, because the electrostatic potential can be averaged owing to the relatively large tip radius $(\simeq 10 \mathrm{~nm})$ compared with the size of $\mathrm{CuPc}$ molecules. $^{21,22}$ However, it can be concluded that the SP of the CuPc molecules was qualitatively higher than that of the $\mathrm{Au}(111)$ substrate.

The molecular-scale SP contrast can be discussed in terms of the previous macroscopic studies. Komolov et al. reported in situ work function measurement on $\mathrm{CuPc}$ films deposited on Au substrates by total current electron spectroscopy using a low-energy electron beam, and showed that the work function of the CuPc films was decreased from 5.1 to $4.5 \pm 0.1 \mathrm{eV}$ as the thickness of the films was increased. ${ }^{23}$ Peisert et al. ${ }^{24,25}$ also reported the negative vacuum level shifts on $\mathrm{CuPc}$ thin films on $\mathrm{Au}$ substrates using ultraviolet photoelectron spectroscopy. These studies strongly suggest the existence of a dipole moment from the Au substrates to the $\mathrm{CuPc}$ molecules, and the present SP measurements agreed well with their results. The origin of the interfacial dipole moment between molecules and metals has been suggested as follows; charge transfer (cation/anion formation), concentration of electrons in the molecule, rearrangement of the electron cloud at the metal surface, strong chemical interaction between the molecules and the metal, existence of interface state, and permanent dipole of molecules. ${ }^{1}$ Peisert et al. also performed x-ray photoelectron spectroscopy on the $\mathrm{CuPc}-\mathrm{Au}$ systems and showed that no chemical interaction occurred on the interfaces and that no $\mathrm{CuPc}$ molecules were cationized. $^{25}$ Their result suggests that the CuPc molecules were physically adsorbed on the Au surfaces. It was considered that the present FM-AFM studies support this discussion. In the case of chemical-adsorption systems [e.g., alkanethiols on $\mathrm{Au}(111)],{ }^{26}$ the reconstructed structures of $\mathrm{Au}(111)$ surfaces disappeared. On the other hand, as shown in Fig. 2(a), it was recognized even on the $\mathrm{CuPc}$ monolayer, which indicated that the interaction between $\mathrm{CuPc}$ and $\mathrm{Au}$ was relatively weak (no chemical interaction). In addition, $\mathrm{CuPc}$ is a nonpolar molecule. Considering these discussions, the origins of the interfacial dipole moment can be suggested as the concentration of electrons in the molecules and/or rearrangement of the electron cloud at the Au surface. Both the origins lead to the positive SP shift (the negative vacuum level shift). The present results agreed well with previous macroscopic studies and suggested that KFM can be used for detecting a single molecular dipole moment on organic/metal interfaces.

\section{CONCLUSION}

We investigated $\mathrm{CuPc}$ monolayers and $\mathrm{CoPc}$ monolayers on the $\mathrm{Au}(111)$ surfaces by FM-AFM. The CuPc molecules formed square-lattice structures and the $\mathrm{CoPc}$ molecules formed square-lattice structures and quasihexagonally packed structures. The submolecular-resolution topographic imaging was achieved on the square-lattice regions of both the monolayers, where the individual molecules were imaged like a four-leaf clover. The center of the CuPc molecules and $\mathrm{CoPc}$ molecules were imaged as a hole and a flat, respectively, while they have almost similar geometry. Their total electron density and molecular orbitals were calculated and the spatial distributions of HOMO and LUMO corresponded well with the obtained FM-AFM contrast. The present study 
indicated that a chemical interaction owing to the hybridization of molecular orbitals between a tip front atom and a sample molecule was a major origin of the submolecularscale contrast and that spatial distribution of chemically active molecular orbitals such as HOMO and LUMO can be imaged by using FM-AFM.

In addition, a molecular-resolution SP image was obtained on the CuPc monolayer by KFM utilizing FM-AFM. The SP of the CuPc molecules was higher than that of the intermolecular regions by approximately $30 \mathrm{mV}$. This result qualitatively agreed well with the previous macroscopic SP studies, and it strongly suggested that the molecular-scale arrangements of electric dipole moments on organic/metal interfaces can be imaged using KFM.

\section{ACKNOWLEDGMENTS}

This work was supported by a Grant-in-Aid for Scientific Research S (Grant No. 19106001) and Global COE Program from Japan Society for the Promotion of Science.

${ }^{1}$ H. Ishii, K. Sugiyama, E. Ito, and K. Seki, Adv. Mater. (Weinheim, Ger.) 11, 605 (1999).

${ }^{2}$ C. D. Dimitrakopoulos and P. R. L. Malenfant, Adv. Mater. (Weinheim, Ger.) 14, 99 (2002).

${ }^{3}$ S. Morita, R. Wiesendanger, and E. Meyer, Noncontact Atomic Microscopy (Springer, Berlin, Heidelberg, 2002) (and references therein).

${ }^{4}$ F. J. Giessibl, S. Hambacher, H. Bielefeldt, and J. Mannhart, Science 289, 422 (2000).

${ }^{5}$ H. Onishi, A. Sasahara, H. Uetsuka, and T. Ishibashi, Appl. Surf. Sci. 188,
257 (2002).

${ }^{6}$ T. Ichii, T. Fukuma, K. Kobayashi, H. Yamada, and K. Matsushige, Jpn. J. Appl. Phys., Part 1 43, 4545 (2004).

${ }^{7}$ S. Kitamura and M. Iwatsuki, Appl. Phys. Lett. 72, 3154 (1998).

${ }^{8}$ C. W. Tang, Appl. Phys. Lett. 48, 183 (1986).

${ }^{9}$ Z. Bao, A. J. Lovinger, and A. Dodabalepur, Appl. Phys. Lett. 69, 3066 (1996).

${ }^{10}$ J. Zhang, J. Wang, H. Wang, and D. Yan, Appl. Phys. Lett. 84, 142 (2004).

${ }^{11}$ K. Kobayashi, H. Yamada, H. Itoh, T. Horiuchi, and K. Matsushige, Rev. Sci. Instrum. 72, 4383 (2001)

${ }^{12}$ T. Fukuma, K. Kobayashi, H. Yamada, and K. Matshushige, J. Appl. Phys. 95, 4742 (2004)

${ }^{13}$ T. Yoda, T. Ichii, T. Fukuma, K. Kobayashi, H. Yamada, and K. Matshushige, Jpn. J. Appl. Phys., Part 1 43, 4691 (2004).

${ }^{14}$ Ch. Wöll, S. Chiang, R. J. Wilson, and P. H. Lippel, Phys. Rev. B 39, 7988 (1989).

${ }^{15}$ I. Chizhov, G. Scoles, and A. Kahn, Langmuir 16, 4358 (2000).

${ }^{16}$ R. D. Shannon, Acta Crystallogr., Sect. A: Cryst. Phys., Diffr., Theor. Gen. Crystallogr. 32, 751 (1976).

${ }^{17}$ K. W. Hipps, X. Lu, X. D. Wang, and U. Mazur, J. Phys. Chem. 100, 11207 (1996).

${ }^{18}$ X. Lu, K. W. Hipps, X. D. Wang, and U. Mazur, J. Am. Chem. Soc. 118, 7197 (1996)

${ }^{19}$ R. Pérez, M. C. Payne, I. Stich, and K. Terakura, Phys. Rev. Lett. 78, 678 (1997).

${ }^{20}$ K. Tagami, N. Sasaki, and M. Tsukada, J. Phys. Soc. Jpn. 69, 3937 (2000).

${ }^{21}$ S. Ono and T. Takahashi, Jpn. J. Appl. Phys., Part 1 43, 4639 (2004).

${ }^{22}$ U. Zerweck, C. Loppacher, T. Otto, S. Grafström, and L. M. Eng, Phys. Rev. B 71, 125424 (2005).

${ }^{23}$ A. Komolov and P. J. Møller, Appl. Surf. Sci. 244, 573 (2005).

${ }^{24}$ H. Peisert, M. Knupfer, and J. Fink, Surf. Sci. 515, 491 (2002).

${ }^{25}$ H. Peisert, M. Knupfer, T. Schwiger, J. M. Auerhammer, M. S. Golden, and J. Fink, J. Appl. Phys. 91, 4872 (2002).

${ }^{26}$ G. E. Poirier, W. P. Fitts, and J. M. White, Langmuir 17, 1176 (2001). 